\title{
Evidence for a New Resonance from Polarized Neutron-Proton Scattering
}

P. Adlarson, ${ }^{1}$ W. Augustyniak, ${ }^{2}$ W. Bardan, ${ }^{3}$ M. Bashkanov, ${ }^{4,5}$ F.S. Bergmann, ${ }^{6}$ M. Berłowski,${ }^{7}$ H. Bhatt,${ }^{8}$

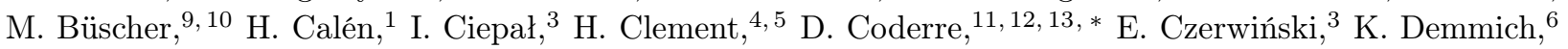

E. Doroshkevich, ${ }^{4,5}$ R. Engels, ${ }^{11,12}$ A. Erven,${ }^{14,12}$ W. Erven, ${ }^{14,12}$ W. Eyrich, ${ }^{15}$ P. Fedorets,,${ }^{11,12,16}$

K. Föhl, ${ }^{17}$ K. Fransson, ${ }^{1}$ F. Goldenbaum, $,{ }^{11}, 12$ P. Goslawski, ${ }^{6}$ A. Goswami, ${ }^{11,12,18}$ K. Grigoryev,${ }^{12,19,20}$

C.-O. Gullström, ${ }^{1}$ F. Hauenstein, ${ }^{15}$ L. Heijkenskjöld, ${ }^{1}$ V. Hejny,${ }^{11,12}$ M. Hodana,${ }^{3}$ B. Höistad,${ }^{1}$ N. Hüsken,${ }^{6}$

A. Jany, ${ }^{3}$ B.R. Jany, ${ }^{3}$ L. Jarczyk,${ }^{3}$ T. Johansson, ${ }^{1}$ B. Kamys, ${ }^{3}$ G. Kemmerling, ${ }^{14,}{ }^{12}$ F.A. Khan, ${ }^{11,12}$

A. Khoukaz, ${ }^{6}$ D.A. Kirillov, ${ }^{21}$ S. Kistryn, ${ }^{3}$ H. Kleines,${ }^{14,12}$ B. Kłos,${ }^{22}$ M. Krapp,${ }^{15}$ W. Krzemien,${ }^{3}$

P. Kulessa, ${ }^{23}$ A. Kupść,,${ }^{1,7}$ K. Lalwani, ${ }^{8}$, D. Lersch, ${ }^{11,12}$ B. Lorentz, ${ }^{11,12}$ A. Magiera, ${ }^{3}$ R. Maier, ${ }^{11,12}$

P. Marciniewski, ${ }^{1}$ B. Mariański, ${ }^{2}$ M. Mikirtychiants, ${ }^{11}, 12,13,{ }^{20}$ H.-P. Morsch, ${ }^{2}$ P. Moskal, ${ }^{3}$ H. Ohm,,${ }^{11,12}$

I. Ozerianska, ${ }^{3}$ E. Perez del Rio, ${ }^{4,5}$ N.M. Piskunov, ${ }^{21}$ P. Podkopal,${ }^{3}$ D. Prasuhn,${ }^{11,12}$ A. Pricking,${ }^{4,5}$

D. Pszczel,${ }^{1,7}$ K. Pysz, ${ }^{23}$ A. Pyszniak,,${ }^{1,3}$ C.F. Redmer,, , J. Ritman, ${ }^{11,12,13}$ A. Roy, ${ }^{18}$ Z. Rudy, ${ }^{3}$

S. Sawant,${ }^{8,11,12}$ S. Schadmand, ${ }^{11,12}$ T. Sefzick, ${ }^{11,12}$ V. Serdyuk, ${ }^{11,12}$ V. Serdyuk, ${ }^{11,12,24}$ R. Siudak, ${ }^{23}$

T. Skorodko, ${ }^{4,5}$ M. Skurzok, ${ }^{3}$ J. Smyrski, ${ }^{3}$ V. Sopov ${ }^{16}$ R. Stassen,,${ }^{11,12}$ J. Stepaniak, ${ }^{7}$ E. Stephan, ${ }^{22}$

G. Sterzenbach, ${ }^{11,12}$ H. Stockhorst, ${ }^{11,12}$ H. Ströher, ${ }^{11,12}$ A. Szczurek, ${ }^{23}$ A. Täschner, ${ }^{6}$ A. Trzciński, ${ }^{2}$

R. Varma ${ }^{8}$ G.J. Wagner, ${ }^{4,5}$ M. Wolke, ${ }^{1}$ A. Wrońska, ${ }^{3}$ P. Wüstner, ${ }^{14,12}$ P. Wurm, ${ }^{11,12}$ A. Yamamoto, ${ }^{25}$

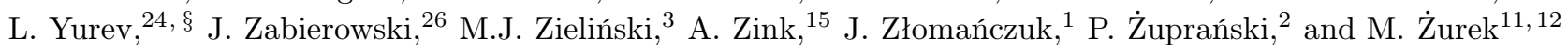

(WASA-at-COSY Collaboration)

\section{R. L. Workman, ${ }^{27}$ W. J. Briscoe,${ }^{27}$ and I. I. Strakovsky ${ }^{27}$}

(SAID Data Analysis Center)

${ }^{1}$ Division of Nuclear Physics, Department of Physics and Astronomy, Uppsala University, Box 516, 75120 Uppsala, Sweden

${ }^{2}$ Department of Nuclear Physics, National Centre for Nuclear Research, ul. Hoza 69, 00-681, Warsaw, Poland

${ }^{3}$ Institute of Physics, Jagiellonian University, ul. Reymonta 4, 30-059 Kraków, Poland

${ }^{4}$ Physikalisches Institut, Eberhard-Karls-Universität Tübingen, Auf der Morgenstelle 14, 72076 Tübingen, Germany

${ }^{5}$ Kepler Center for Astro and Particle Physics, University of Tübingen, Auf der Morgenstelle 14, 72076 Tübingen, Germany

${ }^{6}$ Institut für Kernphysik, Westfälische Wilhelms-Universität Münster, Wilhelm-Klemm-Str. 9, 48149 Münster, Germany

${ }^{7}$ High Energy Physics Department, National Centre for Nuclear Research, ul. Hoza 69, 00-681, Warsaw, Poland

${ }^{8}$ Department of Physics, Indian Institute of Technology Bombay, Powai, Mumbai-400076, Maharashtra, India

${ }^{9}$ Peter Grünberg Institut, Forschungszentrum Jülich, 52425 Jülich, Germany

${ }^{10}$ Institut für Laser- und Plasmaphysik, Heinrich-Heine Universität Düsseldorf, 40225 Düsseldorf, Germany

${ }^{11}$ Institut für Kernphysik, Forschungszentrum Jülich, 52425 Jülich, Germany

${ }^{12}$ Jülich Center for Hadron Physics, Forschungszentrum Jülich, 52425 Jülich, Germany

${ }^{13}$ Institut für Experimentalphysik I, Ruhr-Universität Bochum, Universitätsstr. 150, 44780 Bochum, Germany

${ }^{14}$ Zentralinstitut für Engineering, Elektronik und Analytik, Forschungszentrum Jülich, 52425 Jülich, Germany

${ }^{15}$ Physikalisches Institut, Friedrich-Alexander-Universität

Erlangen-Nürnberg, Erwin-Rommel-Str. 1, 91058 Erlangen, Germany

${ }^{16}$ Institute for Theoretical and Experimental Physics, State Scientific Center of

the Russian Federation, Bolshaya Cheremushkinskaya 25, 117218 Moscow, Russia

${ }^{17}$ II. Physikalisches Institut, Justus-Liebig-Universität Gießen, Heinrich-Buff-Ring 16, 35392 Giessen, Germany

${ }^{18}$ Department of Physics, Indian Institute of Technology Indore, Khandwa Road, Indore-452017, Madhya Pradesh, India

${ }^{19}$ III. Physikalisches Institut B, Physikzentrum, RWTH Aachen, 52056 Aachen, Germany

${ }^{20}$ High Energy Physics Division, Petersburg Nuclear Physics Institute,

Orlova Rosha 2, Gatchina, Leningrad district 188300, Russia

${ }^{21}$ Veksler and Baldin Laboratory of High Energiy Physics, Joint

Institute for Nuclear Physics, Joliot-Curie 6, 141980 Dubna, Russia

${ }^{22}$ August Chetkowski Institute of Physics, University of Silesia, Uniwersytecka 4, 40-007, Katowice, Poland

${ }^{23}$ The Henryk Niewodniczański Institute of Nuclear Physics, Polish

Academy of Sciences, 152 Radzikowskiego St, 31-342 Kraków, Poland

${ }^{24}$ Dzhelepov Laboratory of Nuclear Problems, Joint Institute for Nuclear Physics, Joliot-Curie 6, 141980 Dubna, Russia

${ }^{25}$ High Energy Accelerator Research Organisation KEK, Tsukuba, Ibaraki 305-0801, Japan

${ }^{26}$ Department of Cosmic Ray Physics, National Centre for Nuclear Research, ul. Uniwersytecka 5, 90-950 Łódż, Poland

${ }^{27}$ Data Analysis Center at the Institute for Nuclear Studies, Department of

Physics, The George Washington University, Washington, D.C. 20052, U.S.A.

(Dated: April 18, 2018)

Exclusive and kinematically complete high-statistics measurements of quasifree polarized $\vec{n} p$ scattering have been performed in the energy region of the narrow resonance-like structure $d^{*}$ with 
$I\left(J^{P}\right)=0\left(3^{+}\right), M \approx 2380 \mathrm{MeV}$ and $\Gamma \approx 70 \mathrm{MeV}$ observed recently in the double-pionic fusion channels $p n \rightarrow d \pi^{0} \pi^{0}$ and $p n \rightarrow d \pi^{+} \pi^{-}$. The experiment was carried out with the WASA detector setup at COSY having a polarized deuteron beam impinged on the hydrogen pellet target and uti-

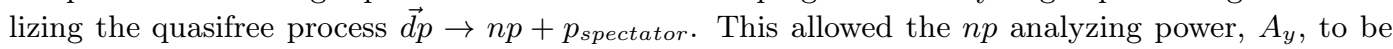
measured over a broad angular range. The obtained $A_{y}$ angular distributions deviate systematically from the current SAID SP07 NN partial-wave solution. Incorporating the new $A_{y}$ data into the SAID analysis produces a pole in the ${ }^{3} D_{3}-{ }^{3} G_{3}$ waves in support of the $d^{*}$ resonance hypothesis.

PACS numbers: 13.75.Cs, 13.85.Dz, 14.20.Pt

\section{INTRODUCTION}

Recent exclusive and kinematically complete measurements of the basic double-pionic fusion reactions $p n \rightarrow$ $d \pi^{0} \pi^{0}$ and $p n \rightarrow d \pi^{+} \pi^{-}$revealed a narrow resonancelike structure in the total cross section [1-3] at a mass $M \approx 2380 \mathrm{MeV}$ with a width of $\Gamma \approx 70 \mathrm{MeV}$, which is consistent with a $I\left(J^{P}\right)=0\left(3^{+}\right)$assignment [2]. Additional evidence for this structure has recently been found in the $p n \rightarrow p p \pi^{0} \pi^{-}$reaction [4], where it was denoted by $d^{*}$, following the notation associated with the so-called "inevitable dibaryon" [5].

If the observed resonance-like structure truly constitutes an $s$-channel resonance in the neutron-proton system, then it must be seen in the observables of elastic $n p$ scattering. In Ref. [6] this resonance effect in $n p$ scattering has been estimated. There it was shown that a noticeable effect should appear in the analyzing power $A_{y}$, since this observable is composed only of interference terms between partial waves, thus being most sensitive to small changes in the partial waves.

For the analyzing power, there exist data only below and above the resonance region. These data sets, at $T_{n}=1.095 \mathrm{GeV}(\sqrt{s}=2.36 \mathrm{GeV})\left[7\right.$, 8] and $T_{n}=1.27$ $\mathrm{GeV}(\sqrt{s}=2.43 \mathrm{GeV})[9,10]$, exhibit very similar angular distributions. This gap in the existing measurements of $A_{y}$ has motivated the present study.

\section{EXPERIMENT}

We have measured the energy dependence of polarized $\vec{n} p$ elastic scattering in the quasifree mode. The experiment was carried out with the WASA detector [11, 12] at COSY (FZ Jülich), using a polarized deuteron beam with an energy of $T_{d}=2.27 \mathrm{GeV}$ impinging on the WASA hydrogen pellet target. With this setup, a full energy coverage of the conjectured resonance was obtained. Note that we observe here the quasi-free scattering process $\overrightarrow{d p} \rightarrow n p+p_{\text {spectator }}$ in inverse kinematics, which allows a detection of the fast spectator proton in the forward detector of WASA.

Since we deal here with events originating from channels with large cross section, the trigger was solely requesting one hit in the first layer of the forward range hodoscope. This hit could originate from either a charged particle or a neutron. For the case of quasifree $n p$ scattering, this defines three event classes, each having the spectator proton appearing in the forward detector:

- scattered proton and scattered neutron both detected in the central detector, covering the neutron angle region $31^{\circ}<\Theta_{n}^{c m}<129^{\circ}$,

- scattered proton detected in the forward detector, with the scattered neutron being unmeasured, covering $132^{\circ}<\Theta_{n}^{c m}<178^{\circ}$ and

- scattered proton detected in the central detector, with the neutron being unmeasured, covering the angular range $30^{\circ}<\Theta_{n}^{c m}<41^{\circ}$.

Combining events, nearly the full range of neutron scattering angles could be covered.

Since, through the use of the inverse kinematics, the spectator proton is in the beam particle, the deuteron, the spectator is very fast. This allows its detection in the forward detector. By reconstruction of its kinetic energy and its direction the full four-momentum of the spectator proton has been determined.

Similarly, the four-momentum of the actively scattered proton has been obtained from its track information in either the forward or central detector (in the latter case the energy information was not retrieved).

Therefore, we have reconstructed the full event, including the four-momentum of the unmeasured neutron, and even have one overconstraint in the subsequent kinematic fit, when the neutron has not been measured explicitly.

In the case where the neutron has been detected by a hit in the calorimeter (composed of $1012 \mathrm{CsI}(\mathrm{Na})$ crystals) of the central detector - associated with no hit in the preceding plastic scintillator barrel, the directional information of the scattered neutron has also been obtained. Therefore, these events have undergone a kinematic fit with two overconstraints.

In order to avoid a distortion of the beam polarization, the magnetic field of the solenoid in the central detector was switched off. The measurements were carried out with cycles of the beam polarization "up", "down" and unpolarized (originating from the same polarized source), where "up" and "down" refers to a horizontal scattering plane. We verified that the beam, originating from the polarized source, indeed was unpolarized when using it in 
its "unpolarized" mode. This was accomplished by comparing the azimuthal angular dependence of the scattered events to that obtained through the use of a conventional unpolarized source.

The magnitude of the beam polarization was determined and monitored by $d p$ elastic scattering, which was measured in parallel by detecting the scattered deuteron in the forward detector as well as the associated scattered proton in the central detector. The vector and tensor components of the deuteron beam were obtained by fitting our results, for the vector and tensor analyzing power, to those obtained previously at ANL [13] for $T_{d}$ $=2.0 \mathrm{GeV}$ and more recently at COSY-ANKE [14] at $T_{d}=2.27 \mathrm{GeV}$. As a result we obtained beam polarizations of $P_{z}=0.67(2), P_{z z}=0.65(2)$ for "up" and $P_{z}$ $=-0.45(2), P_{z z}=0.17(2)$ for "down". The vector polarization of the beam, for quasifree scattering, has been checked by quasifree $p p$ scattering. This was also measured in parallel by detecting one of the protons in the forward detector and the other one in the central detector, in addition, checking their angular correlation for elastic events. Our results for the quasifree $p p$ analyzing power are in quantitative agreement both with the EDDA measurements [15] of free $p p$ scattering and with the current SAID phase shift solution SP07 [16].

Since we have measurements with spin "up", "down" and unpolarized, the vector analyzing power can be derived in three different ways, by using each two of the three spin orientations. All three methods should give identical results. Differences may be taken as an estimate of systematic uncertainties which are added quadratically to the statistical ones to give the total uncertainties plotted in Figs. 1,2 and 4.

The momentum distribution of the observed spectator proton, in the elastic $n p$ scattering process, agrees with Monte Carlo simulations of the proton momentum distribution in the deuteron filtered by the acceptance of the WASA detector. In order to assure a quasi-free process, we omit events with spectator momenta larger than 0.16 $\mathrm{GeV} / \mathrm{c}$ (in the deuteron rest system) as done in previous work [2, 3$]$.

\section{RESULTS AND DISCUSSION}

Due to the Fermi motion of the nucleons bound in the beam deuteron, the measurement of the quasi-free $n p$ scattering process covers a range of energies in the $n p$ system. Meaningful statistics could be collected for the range of $n p$ center-of-mass energies $2.36<\sqrt{s}<2.41$ $\mathrm{GeV}$ corresponding to $T_{n}=1.10-1.20 \mathrm{GeV}$. First, we show the data (solid circles) in Fig. 1 without selecting specific $n p$ center-of-mass energies, i.e. without accounting for the spectator momentum. Hence this data set corresponds to the weighted average over the covered interval of $\sqrt{s}$. The solid line represents the current SAID

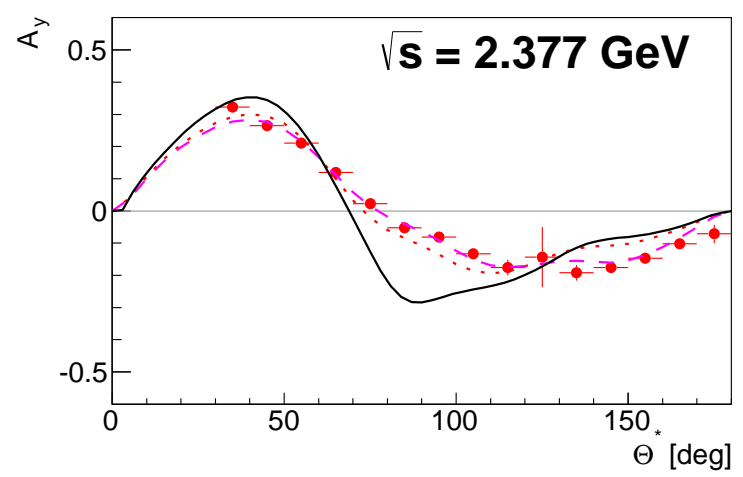

FIG. 1. (Color online) Angular distribution of the $n p$ analyzing power without consideration of the spectator momentum, corresponding to a weighted average over the measured interval $\sqrt{s}=2.367-2.403 \mathrm{GeV}\left(T_{n}=1.108-1.197 \mathrm{GeV}\right)$ with a centroid at $\sqrt{s}=2.377 \mathrm{GeV}$. The results from this work are shown as solid circles with error bars including both statistical and systematic uncertainties. The solid line represents the SAID SP07 phase shift prediction [16], whereas the dashed (dotted) line gives the result of the new weighted (unweighted) SAID partial-wave solution (see text).

SP07 partial-wave solution [16], whereas dashed and dotted lines give the results of revised SAID partial-wave analyses, including the WASA dataset, as described below. Next, we have taken the measured spectator fourmomentum into account and constructed the effective $\sqrt{s}$ for each event. We thus obtained angular distributions sorted into six $\sqrt{s}$ bins, two of which are shown in Fig. 2 as examples. All of our data deviate strikingly from the SP07 solution.

As a test, the present $A_{y}$ data set was included in the SAID database and the phenomenological approach used in generating the $N N$ partial-wave solution, SP07 [16], was retained. Here we first considered whether the existing form was capable of describing these new $A_{y}$ measurements. One advantage of this approach is that the employed Chew-Mandelstam K-matrix can produce a pole in the complex energy plane without the explicit inclusion of a K-matrix pole in the fit form. Neither the existence of a pole nor the effected partial waves are predetermined. A detailed overview of this formalism is given in Ref.[17].

The fitted $A_{y}$ data were angular distributions at $T_{\mathrm{Lab}}$ values of $1.108,1.125,1.135,1.139,1.156,1.171$, and $1.197 \mathrm{GeV}$. A first attempt to fit this dataset started from the functional form of the current SP07 fit, and only varied the associated free parameters. A $\chi^{2} /$ datum of 1.8 was found for all angular distributions, apart from the one at $1135 \mathrm{MeV}$. This was fairly consistent with the overall $\chi^{2}$ /datum given by the global fit of $n p$ elastic scattering data to $2 \mathrm{GeV}$. However, the set at 1135 $\mathrm{MeV}$ contributed a $\chi^{2} /$ datum of about 25 , having better statistics and a wider angular coverage. 

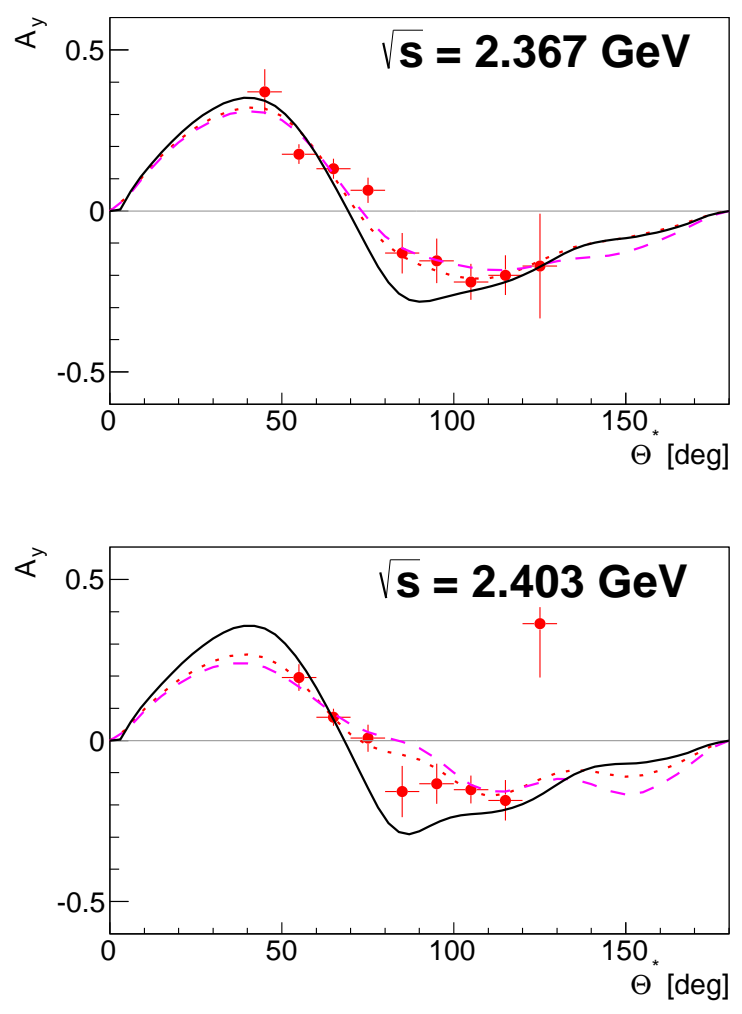

FIG. 2. (Color online) Notation as in Fig. 1, but for $\sqrt{s}$ $=2.367$ (top) and $2.403 \mathrm{GeV}$ (bottom) corresponding to $T_{n}$ $=1.11$ and $1.20 \mathrm{GeV}$. The full symbols denote results from this work taking into account the spectator four-momentum information. For the meaning of the curves see Fig. 1.

The fit parameters are expansion coefficients for the K-matrix elements, which are smooth in energy; either polynomials or basis elements having required left-hand cuts, as described in Ref.[17]. Failing to reproduce the $1135 \mathrm{MeV}$ set, the fit form was scanned to find partial waves for which an added term in the K-matrix expansion produced the most efficient reduction in $\chi^{2}$. The addition of parameters and re-fitting resulted in a rapid variation of the coupled ${ }^{3} D_{3}$ and ${ }^{3} G_{3}$ waves in the vicinity of the problematic $1135 \mathrm{MeV}$ data set.

Some weighting was necessary in this fit, as only a few angular points from the full set were determining the altered energy dependence. The fit was repeated with different weightings for the $1135 \mathrm{MeV} A_{y}$ dataset. Having found a better fit at $1135 \mathrm{MeV}$, a subsequent fit was produced without weighting. These, qualitatively similar, results are compared in the figures.

In Fig. 1 we plot the fit to the $1135 \mathrm{MeV}$ angular distribution from the SP07 prediction (not including the new data), a weighted fit ( errors decreased by a factor of 4 ), and an unweighted fit including the present dataset and using the new fit form.

Resulting changes in the ${ }^{3} D_{3^{-}}{ }^{3} G_{3}$ coupled waves are displayed in Fig. 3. Here the ${ }^{3} D_{3}$ wave obtained a typical resonance-like shape, whereas the ${ }^{3} G_{3}$ wave changed less dramatically. A search of the complex energy plane revealed a pole in the coupled ${ }^{3} D_{3^{-}}{ }^{3} G_{3}$ wave. Other partial waves did not change significantly over the energy range spanned by the new data. Fig. 3 also displays singleenergy solutions, generated from the old SP07 fit. These discrete points are fits to data within narrow energy bins, allowing amplitude variations to produce a best fit to data, and are used to search for systematic deviations from the global fit [17]. In the ${ }^{3} D_{3}$ partial-wave plot near $1135 \mathrm{MeV}$, the new fit appears to agree with these single-energy results much better than SP07.

The fit repeated with different weightings for the new $A_{y}$ data resulted in a variation of the pole position and could be considered a minimal 'error' on its value within the present fit form. In the weighted fits, a pole was located at (2392 - i37) MeV. The re-fit without weighting produced a pole with $(2385$ - i39) $\mathrm{MeV}$. Together with a speed-plot determination we arrive at $(2380 \pm 10-i 40 \pm 5)$ $\mathrm{MeV}$ as our best estimate for the pole position.

From the decomposition of the $n p$ observables into partial-wave amplitudes [18], it follows that the resonance contribution in $A_{y}$ is proportional to the associated Legendre polynomial $P_{3}^{1}\left(\cos \Theta_{n}^{c m}\right)$. $P_{3}^{1}$ is maximal at $\Theta_{n}^{c m}=31.1^{\circ}$ and minimal at $90^{\circ}$. Since at the latter angle the differential cross section is at minimum and much lower than at the former angle, the resonance effect in $A_{y}$ becomes maximal at $\Theta_{n}^{c m}=90^{\circ}$. To check this behavior, we have inspected the energy dependence of $A_{y}$. In order to include a reasonable number of previous measurements, we have chosen the nearby angle $\Theta_{n}^{c m}=83^{\circ} \pm 2^{\circ}$ to be plotted in Fig. 4. The data exhibit a pronounced resonance-like behavior in accordance with the new partial-wave solution - in tendency even somewhat narrower.

\section{SUMMARY AND CONCLUSIONS}

In conclusion, our exclusive and kinematically complete measurement of quasi-free polarized $\vec{n} p$ scattering provides detailed high-statistics data for the analyzing power in the energy range, where previously a narrow resonance-like structure with $I\left(J^{P}\right)=0\left(3^{+}\right)$was observed in the double-pionic fusion to deuterium. A partial-wave analysis including the new $n p$ scattering data exhibits a resonance pole in the coupled ${ }^{3} D_{3}-{ }^{3} G_{3}$ partial waves in accordance with the expectation of a $d^{*}$ resonance structure. This structure has been associated with a bound $\Delta \Delta$ resonance, which could contain a mixture of asymptotic $\Delta \Delta[24]$ and 6-quark, hidden color, configurations [25]. Though less exotic explanations cannot be excluded at the present stage, dibaryon systems matching the mass and width of this dibaryon candidate have been recently successfully generated within 3- 

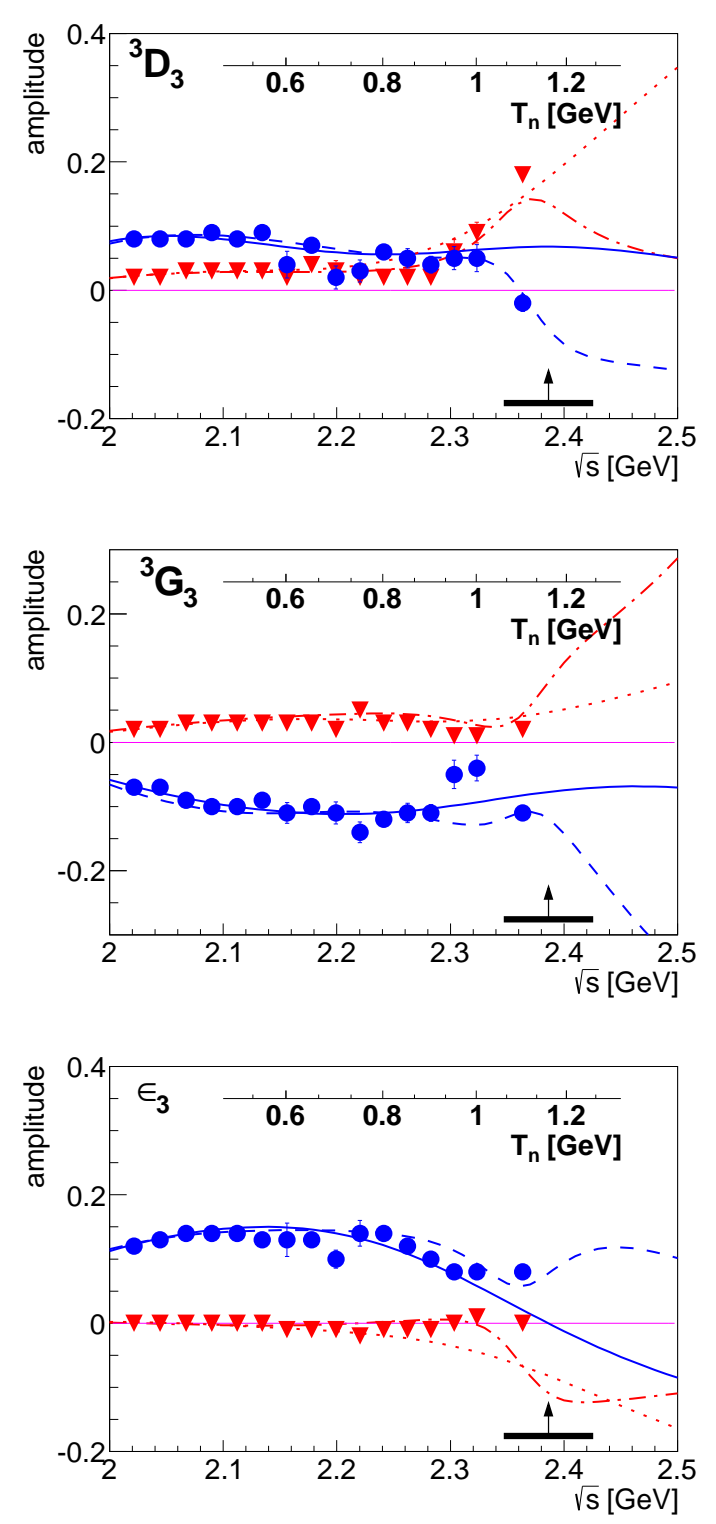

FIG. 3. (Color online) Changes to the (dimensionless) ${ }^{3} D_{3}$ (top) and ${ }^{3} G_{3}$ (middle) partial waves including their mixing amplitude $\epsilon_{3}$ (bottom). Solid (dotted) curves give the real (imaginary) part of the partial-wave amplitudes from SP07, whereas the dashed (dash-dotted) curves represent the new (weighted) solution. Results from previous single-energy fits [16] are shown as solid circles (real part) and inverted triangles (imaginary part). Vertical arrows and horizontal bars indicate mass and width of the resonance (estimated from the pole position).

body [26] and quark model [27] calculations. It should be noted that earlier dibaryon candidates [17] were widely discounted due to their appearance near the $N \Delta$ cut and the possibility of a pseudo-resonance mimicking their behavior. Such complications do not arise here - though we note the existence of a nearby $N N^{*}(1440)$ threshold. However, we are not aware of any mechanism by which

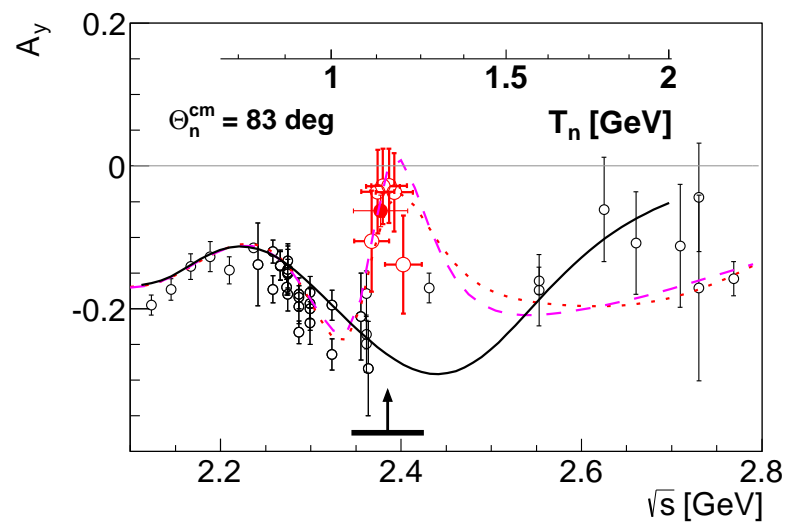

FIG. 4. (Color online) Energy dependence of the $n p$ analyzing power at $\Theta_{n}^{c m}=83^{\circ}$. The solid symbols denote the results from this work, the open symbols those from previous work [7 9, 19 23]. For the meaning of the curves see Fig. 1. Vertical arrow and horizontal bar indicate mass and width of the resonance (estimated from the pole position).

the very broad Roper resonance could induce the narrow resonance structure considered here.

Finally, we note that the new partial-wave solution improves also the description of total cross section data as well as polarization observables obtained at ANKE [28] in the resonance region. A full account of the new results will be given in an extended forthcoming paper.

We acknowledge valuable discussions with J. Haidenbauer, C. Hanhart, A. Kacharava and C. Wilkin on this issue. This work has been supported by BMBF, Forschungszentrum Jülich (COSY-FFE), the U.S. Department of Energy Grant DE-FG0299ER41110, the Polish National Science Centre (grant No. 2011/03/B/ST2/01847) and the Foundation for Polish Science (MPD).

* present address: Albert Einstein Center for Fundamental Physics, University of Bern, Sidlerstrasse 5, 3012 Bern, Switzerland

${ }^{\dagger}$ present address: Department of Physics and Astrophysics, University of Delhi, Delhi-110007, India

$\ddagger$ present address: Institut für Kernphysik, Johannes Gutenberg-Universität Mainz, Johann-Joachim-Becher Weg 45, 55128 Mainz, Germany

$\S$ present address: Department of Physics and Astronomy, University of Sheffield, Hounsfield Road, Sheffield, S3 7RH, United Kingdom

[1] M. Bashkanov et al., Phys. Rev. Lett. 102, 052301 (2009).

[2] P. Adlarson et al., Phys. Rev. Lett. 106, 242302 (2011).

[3] P. Adlarson et al., Phys. Lett. B 721, 229 (2013).

[4] P. Adlarson et al., Phys. Rev. C 88, 055208 (2013).

[5] T. Goldman et al., Phys. Rev. C 39, 1889 (1989). 
[6] A. Pricking, M. Bashkanov, H. Clement, arXiv:1310.5532 [nucl-ex].

[7] J. Ball et al., Nucl. Phys. A 559, 489 (1993).

[8] A. de Lesquen et al., Eur. Phys. J. C 11, 69 (1999).

[9] Y. Makdisi et al., Phys. Rev. Lett. 45, 1529 (1980).

[10] R. Diebold et al., Phys. Rev. Lett. 35, 632 (1975).

[11] Chr. Bargholtz et al., Nucl. Inst. Meth. A 594, 339 (2008).

[12] H. H. Adam et al., arxiv: nucl-ex/0411038

[13] M. Haji-Saied et al., Phys. Rev. C 36, 2010 (1987).

[14] D. Mchedlishvili, Internal ANKE report 28 (2013).

[15] M. Altmeier et al., Eur. Phys. J. A 23, 351 (2005).

[16] SAID data base http://gwdac.phys.gwu.edu/; R. A. Arndt et al., Phys. Rev. C 76, 025209 (2007).

[17] R.A. Arndt, J.S. Hyslop, L.D. Roper, Phys. Rev. D35, 128 (1987).
[18] R. A. Arndt et al., Phys. Rev. D 28, 97 (1983).

[19] C. R. Newsom et al., Phys. Rev. C 39, 965 (1989).

[20] J. Arnold et al., Eur. Phys. J. C 17, 67 (2000).

[21] J. Ball et al., Nucl. Phys. B 286, 635 (1987).

[22] M. W. McNaughton et al., Phys. Rev. C 48, 256 (1993); 53, 1092 (1996).

[23] G. Glass et al., Phys. Rev. C 47, 1369 (1993).

[24] F. J. Dyson, N.-H. Xuong, Phys. Rev. Lett. 13, 815 (1964).

[25] M. Bashkanov, S. Brodsky, H. Clement, Phys. Lett. B727, 438 (2013).

[26] A. Gal, H. Garcilazo, Phys. Rev. Lett. 111, 172301 (2013) and Nucl. Phys. A, in press (DOI: 10.1016/j.nuclphysa.2014.02.019).

[27] H. Huang, J. Ping, F. Wang, Phys. Rev. C89, 034001 (2014).

[28] D. Mchedlishvili et al., Eur. Phys. J. A 49, 49 (2013). 